OPEN ACCESS

Edited by:

Dion Dickman,

University of Southern California,

United States

Reviewed by:

Christian Wegener,

University of Würzburg, Germany

Norio K. Ishida,

National Institute of Advanced Industrial Science and Technology,

Japan

Adam Michael Reitzel,

University of North Carolina at

Charlotte, United States

*Correspondence:

Emi Nagoshi

emi.nagoshi@unige.ch

Received: 20 June 2017 Accepted: 26 September 2017 Published: 12 October 2017

Citation:

Sabado V, Vienne L and Nagoshi E (2017) Evaluating the Autonomy of the Drosophila Circadian Clock in Dissociated Neuronal Culture. Front. Cell. Neurosci. 11:317. doi: 10.3389/fncel.2017.00317

\section{Evaluating the Autonomy of the Drosophila Circadian Clock in Dissociated Neuronal Culture}

\author{
Virginie Sabado, Ludovic Vienne and Emi Nagoshi* \\ Department of Genetics and Evolution, Sciences III, University of Geneva, Geneva, Switzerland
}

Circadian behavioral rhythms offer an excellent model to study intricate interactions between the molecular and neuronal mechanisms of behavior. In mammals, pacemaker neurons in the suprachiasmatic nucleus (SCN) generate rhythms cell-autonomously, which are synchronized by the network interactions within the circadian circuit to drive behavioral rhythms. However, whether this principle is universal to circadian systems in animals remains unanswered. Here, we examined the autonomy of the Drosophila circadian clock by monitoring transcriptional and post-transcriptional rhythms of individual clock neurons in dispersed culture with time-lapse microscopy. Expression patterns of the transcriptional reporter show that CLOCK/CYCLE (CLK/CYC)-mediated transcription is constantly active in dissociated clock neurons. In contrast, the expression profile of the post-transcriptional reporter indicates that PERIOD (PER) protein levels fluctuate and $\sim 10 \%$ of cells display rhythms in PER levels with periods in the circadian range. Nevertheless, PER and TIM are enriched in the cytoplasm and no periodic PER nuclear accumulation was observed. These results suggest that repression of CLK/CYC-mediated transcription by nuclear PER is impaired, and thus the negative feedback loop of the molecular clock is incomplete in isolated clock neurons. We further demonstrate that, by pharmacological assays using the non-amidated form of neuropeptide pigment-dispersing factor (PDF), which could be specifically secreted from larval LNvs and adult s-LNvs, downstream events of the PDF signaling are partly impaired in dissociated larval clock neurons. Although non-amidated PDF is likely to be less active than the amidated one, these results point out the possibility that alteration in PDF downstream signaling may play a role in dampening of molecular rhythms in isolated clock neurons. Taken together, our results suggest that Drosophila clocks are weak oscillators that need to be in the intact circadian circuit to generate robust 24-h rhythms.

Keywords: Drosophila, circadian rhythms, time-lapse imaging, non-cell autonomous, dispersed culture, fluorescent circadian reporter

\section{INTRODUCTION}

Virtually all organisms have circadian clocks, which emerged as an adaptation strategy for life on Earth. Circadian rhythms are synchronized (entrained) to environmental cues, e.g., light-dark cycles (LD) and warm-cool cycles, thereby allowing organisms to anticipate and prepare for daily and seasonal changes. A fundamental property of circadian rhythms is their persistence in the 
absence of inputs, which indicates the existence of an endogenous clock that can free-run with a period of $\sim 24 \mathrm{~h}$ (Zhang and Emery, 2012; Granados-Fuentes and Herzog, 2013).

Some unicellular organisms exhibit circadian rhythms autonomously (Webb and Oates, 2016). In multicellular organisms, clock cells are found in many tissues, where rhythms are synchronized directly by external cues or coordinated by a central clock, such as the SCN in mammals. Different studies using reporters of the molecular clock have shown that circadian rhythmicity is maintained in individual SCN neurons in organotypic culture (Yamaguchi et al., 2003; Yoo et al., 2004; Fang et al., 2007; Liu et al., 2007). In dissociated SCN culture, high-amplitude circadian rhythms persist in most but not all neurons (Liu et al., 2007). The proportion of robustly cycling neurons further decreases and the rhythms are more desynchronized in low-density culture (Webb et al., 2009). It has been concluded that, although fundamentally cell-autonomous, SCN neurons require intercellular coupling to exhibit robust circadian rhythms (Ko et al., 2010). This is in stark contrast to mammalian fibroblasts, which generate robust, self-sustaining rhythms regardless of density and culture conditions (Nagoshi et al., 2004; Welsh et al., 2004). Thus, central clocks are less robust than peripheral clocks at the single cell level in mammals. Importantly, however, damped oscillators respond more readily to each other and to the environmental cycle to achieve fast synchronization (Ko et al., 2010; Webb and Oates, 2016). These characteristics are particularly advantageous for the central pacemaker.

In adult Drosophila, $\sim 150$ clock neurons form a circuit that orchestrates the circadian behavior. The circadian circuitry exists also in the larval brain, which is composed of three groups of clock neurons, i.e., five ventral Lateral Neurons (LNvs), two Dorsal Neurons 1 (DN1s) and two Dorsal Neurons 2 (DN2s) (reviewed in Helfrich-Forster, 2005). Larval circuitry is known to govern circadian behavior in response to light (Malpel et al., 2004; Mazzoni et al., 2005). In the core feedback loop of each clock neuron, the transcription of period (per) and timeless (tim) is activated by CLK/CYC heterodimer through binding to E-boxes located on the promoters of per and tim (Hardin and Panda, 2013). PER and TIM enter the nucleus and inhibit CLK/CYC activity, leading to the repression of their own transcription. Various post-transcriptional and posttranslational regulations, such as PER phosphorylation and TIM degradation, take place to complete the core feedback loop in circa $24 \mathrm{~h}$ (Sheeba et al., 2008; Zhang and Emery, 2012). Light, the main input of the circadian clock, is sensed by photoreceptors and the blue-light photopigment cryptochrome (CRY; Reviewed in Yoshii et al., 2016). Light-activated CRY induces the degradation of TIM and destabilizes PER/TIM heterodimer, leading to the phase resetting or arrhythmicity depending on the light regime (Emery et al., 1998; Stanewsky et al., 1998; Zhang and Emery, 2012). Additional transcriptional feedback loops contribute to the robustness of the core feedback loop. Altogether, these interlocked Transcriptional-Translational Feedback Loops (TTFL) constitute the molecular clock (Sheeba et al., 2008; Hardin, 2011).
To examine the intrinsic properties of Drosophila clock neurons, we recently developed two fluorescent reporters of the molecular clockwork that permit single-cell recording of the transcriptional and post-transcriptional machineries (Sabado et al., 2017). The transcriptional reporter $3 \times 69$ VNP expresses the short-lived yellow fluorescent protein (VNP; VENUS-NLS-PEST) under the control of three tandem repeats of the E-box-containing 69-bp clock regulatory sequence (CRS) of per (Hao et al., 1997, 1999). $3 \times 69$-VNP thus mimics the CLK/CYC-dependent transcriptional rhythms and per mRNA oscillations. PER protein reporter PER-TDT was built through the fusion of per endogenous promoter, two-thirds of the coding sequence of per, the cDNA encoding the red fluorescent protein tandem TOMATO (TDT) and per 3'-UTR. Both reporters are expressed rhythmically in clock neurons in vivo and in brain explant culture with expected phases (Sabado et al., 2017).

In this study, we investigate the state of autonomy of isolated Drosophila clock neurons by taking advantage of the long-term live imaging of molecular clockwork with our fluorescent reporters. The results demonstrate that Drosophila clocks are intrinsically unstable oscillators that rely on intact circuitry to generate robust circadian rhythms.

\section{RESULTS}

Previous works have shown that Drosophila clock neurons in cultured adult brains exhibit circadian molecular rhythms (Sellix et al., 2010; Roberts et al., 2015). By using fluorescent transcriptional reporter $3 \times 69$-VNP and PER protein reporter PER-TDT (Figure 1A, Supplementary Figures 1A,B), we have also demonstrated that molecular rhythms of larval clock neurons in cultured brains are detectable by time-lapse microscopy. Although reporter fluorescence levels varied between animals and among individual neurons, we observed circadian expression of both $3 \times 69$-VNP and PER-TDT in more than $50 \%$ of larval clock neurons (Sabado et al., 2017). These findings unequivocally indicate the ability of individual fly clock neurons to generate rhythms without environmental cues in the isolated brain, where circadian circuit is intact. However, whether fly clock neurons are cell-autonomous oscillators remains to be formally tested.

To this end, we cultured dissociated neurons derived from third instar larval brains in the same medium used for brain explant culture and imaged $3 \times 69-\mathrm{VNP}$ and PER-TDT reporter expression by time-lapse microscopy. Clock neurons expressing $3 \times 69$-VNP or PER-TDT were visualized by co-expressing UAS$m C D 8:: R F P$ with 1982clk-gal4 (labeling all three subtypes), or UAS- $m C D 8::$ Venus with gal1118 (labeling the LNvs), respectively. Images were acquired every $3 \mathrm{~h}$ for $48 \mathrm{~h}$, i.e., with the same interval and duration of time-lapse used to monitor rhythms in cultured brains (Sabado et al., 2017). Surprisingly, the majority of clock neurons displayed a steady increase in $3 \times 69$-VNP levels over time without detectable oscillations. By contrast, PERTDT expression showed fluctuations of varying patterns with $\sim 13 \%$ of the clock neurons displaying rhythms in the circadian range (mean period \pm SEM, $26.143 \pm 1.079 \mathrm{~h}$; Figures 1B,C, 
Supplementary Movies S1, S2). PER-TDT expression profiles of the LNvs and non-LNvs were indistinguishable, and the rhythmic cells were not restricted to the LNvs.

The cultured larval neurons maintained the characteristics of healthy primary neurons (Saad et al., 2012) throughout time-lapse imaging (Supplementary Figure 2). The neurons expressing reporters in dissociated culture also expressed endogenous PER and TIM; their levels did not decrease after live imaging and were not significantly different between wild-type (wt) and $c r y^{0}$ backgrounds (Figures 2A-D). Therefore, the absence of $3 \times 69$-VNP rhythms in dissociated clock neurons is not due to the deterioration of viability, laser illumination or light-mediated activation of CRY but rather reflects the properties of neuronal oscillators. The observation that PER-TDT levels do not linearly increase but fluctuate suggests that certain post-transcriptional regulations contributing to the generation of PER rhythms is still operational in isolated neurons, preventing the over-accumulation of PER despite the constant upregulation of CLK/CYC-mediated transcription. In addition, some transcriptional regulation of per via cisregulatory elements other than CRS is probably also functional in cultured clock neurons, contributing to the fluctuating PER-TDT expression.

To explore the mechanisms leading to these changes in molecular rhythms in isolated neurons, we next analyzed the subcellular localization of endogenous PER and TIM. Interestingly, PER and TIM were detected in both nucleus and cytoplasm but enriched in the cytoplasm of cultured clock neurons. Live imaging did not affect the nuclear/cytoplasmic ratio (Figures 2C,E). Furthermore, consistent with the subcellular localization pattern of endogenous PER, PER-TDT reporter was enriched in the cytoplasm throughout the recording period of live imaging. A small proportion of neurons showed PER-TDT nuclear enrichment sporadically, but without any apparent rhythms (Figure 2F). These results indicate that timed nuclear entry of PER is impaired in dissociated cultured neurons. This finding further suggests that the feedback inhibition of CLK/CYC transcriptional activity by PER may be dysregulated, resulting in a continuous, non-rhythmic CLK/CYC-mediated transcription. This would explain the constant rise of $3 \times 69$-VNP signals in dissociated clock neurons.

Because clock neurons can generate both $3 \times 69$-VNP and PER-TDT rhythms in whole brain culture (Sabado et al., 2017), the disruption of molecular rhythms in dissociated culture may be caused by the lack of intercellular interactions that normally occur in vivo and in brain explants. Network interactions within the circadian circuit are mediated by various neuropeptides and neurotransmitter signaling (Beckwith and Ceriani, 2015). In particular, a number of studies have shown that the neuropeptide pigment-dispersing factor (PDF) is critical for synchronizing and maintaining rhythms of clock neurons (Peng et al., 2003; Helfrich-Forster, 2005; Yoshii et al., 2009; Yao and Shafer, 2014; Beckwith and Ceriani, 2015). Recent works also reported that PDF regulates the phase of neuronal activity rhythms of different clock neuron subgroups (Liang et al., 2016, 2017). We also recently showed that PDF has a nighttime-specific dual role in regulating the molecular clockwork: enhancing CLK/CYC-mediated transcription and stabilizing PER in larval brains. We further demonstrated that the addition of PDF in dissociated culture increases $3 \times 69-\mathrm{VNP}$ signals in clock neurons, which indicates that PDF signaling stimulates CLK/CYC-mediated transcription cell-autonomously (Sabado et al., 2017). However, whether PDF affects PER stability cell-autonomously in dissociated neurons has not been examined.

Therefore, we added $2 \mu \mathrm{M}$ of PDF to the culture medium, a concentration sufficient to upregulate PER-TDT levels in brain explants (Sabado et al., 2017), and monitored PER-TDT expression every $3 \mathrm{~h}$ for subsequent $24 \mathrm{~h}$. Unexpectedly, the addition of PDF did not affect PER-TDT expression patterns of dissociated clock neurons (Figures 3A,B). These results suggest that, in contrast to the effect of PDF on CLK/CYC-mediated transcription, stabilization of PER by PDF does not occur by default in individual clock neurons but requires them to be in an in vivo-like context.

PDF acts through the PDF receptor (PDFR) that belongs to the G protein-coupled receptor family (Hyun et al., 2005; Lear et al., 2005; Mertens et al., 2005). To learn more about the regulation of individual molecular clocks in dissociated neurons by PDF signaling, we next monitored the reporter expression in cultured clock neurons prepared from $p d f r$ null mutants. In this culture, the medium should contain PDF released from the LNvs, whereas PDF/PDFR signaling is absent in clock neurons. PDF has an important presynaptic role in the development of the LNvs (Gorostiza and Ceriani, 2013), which is not affected in this condition. Interestingly, the majority of clock neurons of $p d f r$ mutants did not show a steady increase but fluctuations in $3 \times 69$-VNP levels, although none were circadian (Figure 4A). This observation suggests that there is a persistent stochastic CLK/CYC-mediated transcription in isolated clock neurons in the absence of $\mathrm{PDF} / \mathrm{PDFR}$ signaling, which gives rise to the fluctuating patterns of the $3 \times 69-\mathrm{VNP}$ signal. This finding also indicates that, in $w t$ culture, $\mathrm{PDF}$ is present in the media and constantly upregulates CLK/CYC-mediated transcription, resulting in the steady increase of $3 \times 69-\mathrm{VNP}$ levels. The proportion of cells displaying PER-TDT rhythms was approximately the same between $w t$ and $p d f r(12.5 \%, 28.2 \pm 1.53 \mathrm{~h}$; Figure 4B). This result is concordant with the above finding that PER stability is insensitive to PDF signaling in dissociated neurons (Figures 3A,B).

\section{DISCUSSION}

Here, we examined the state of autonomy of Drosophila clock neurons by monitoring the expression of two circadian fluorescent reporters, the transcriptional reporter $3 \times 69-\mathrm{VNP}$ and PER protein reporter PET-TDT (Sabado et al., 2017), in long-term time-lapse imaging of dispersed clock neurons. Both reporters are expressed but in a non-circadian manner in most cells. $3 \times 69-$ VNP levels exhibit a steady increase, whereas PER-TDT levels fluctuate and do not increase over time. While approximately one-tenth of clock neurons show 
A
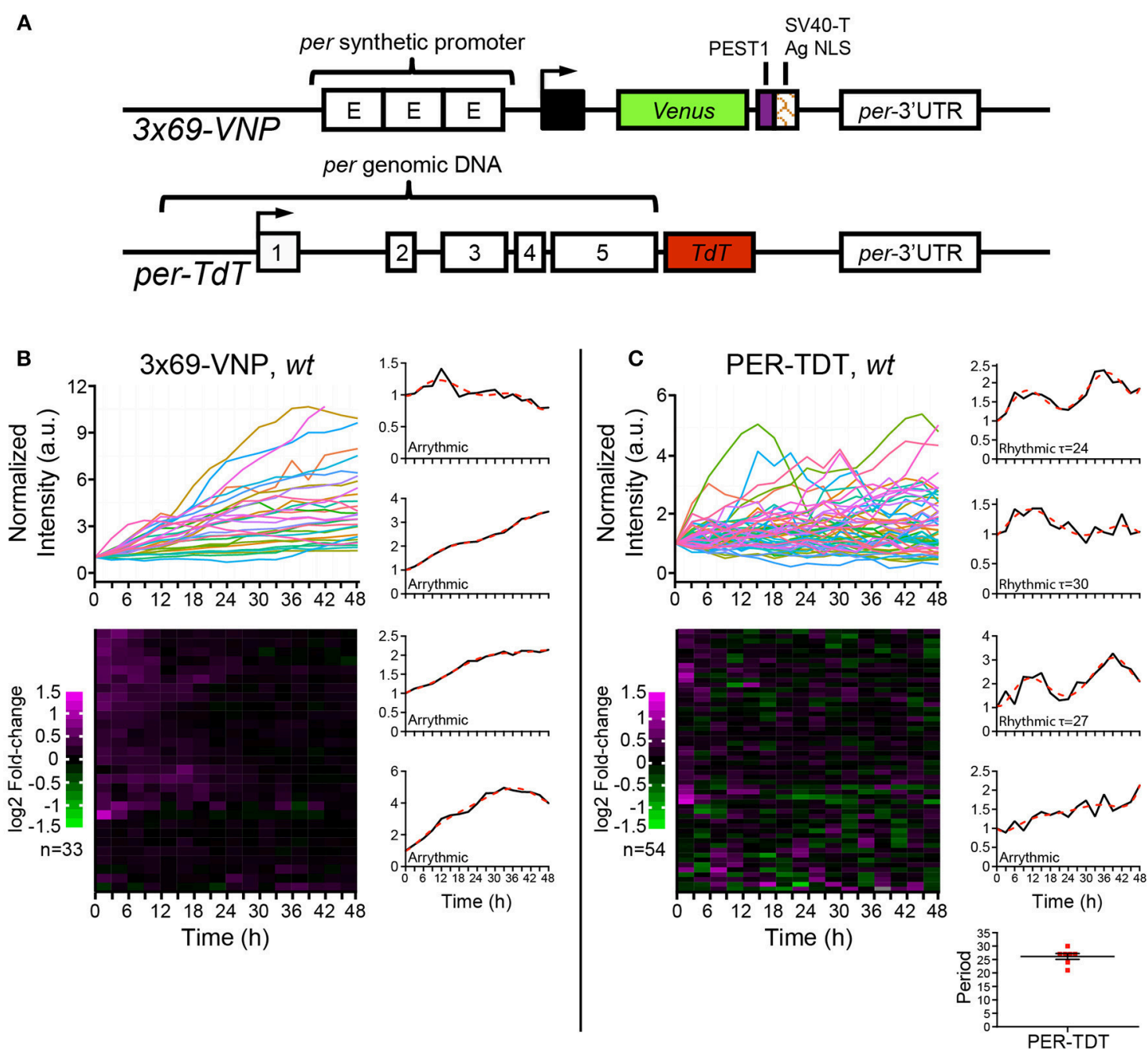

FIGURE 1 | Drosophila neuronal clocks are poorly rhythmic in dissociated culture. (A) Schemes of the 3x69-VNP and PER-TDT reporters (Sabado et al., 2017). (B) $3 \times 69-V N P$ and (C) PER-TDT expression in clock neurons in wild-type (wt) dissociated culture. Graphs indicate relative fluorescence intensity of individual cells normalized to the value at $t=0$. Raster plots indicate the time course of fluorescence intensity fold-change in log2. Each row represents a single cell. Representative single cell traces are shown with the 6th order polynomial trend line (red dotted line). Note that PER-TDT profiles are similar between the LNvs labeled by ga/1118>mCD8::Venus and non-LNv clock neurons, the expression profiles of both cell types are shown without distinction in (B). Bottom right, periods of the 13\% rhythmic neurons in PER-TDT expressing neurons. Mean period \pm SEM was $26.143 \pm 1.079 \mathrm{~h}$. $n$, number of cells.

PER-TDT level fluctuations with periods in the circadian range, no periodic nuclear translocation of PER-TDT was observed. These results suggest that Drosophila circadian clocks are poorly cell-autonomous.

\section{What Underlies the Loss of Rhythms in Dissociated Clock Neurons?}

PER is the major repressor of the core TTFL of the molecular clock. Most clock neurons in culture show cytoplasmic enrichment of PER and TIM (Figures 2C-E), resembling the state of clock neurons around ZT14, a time when CLK/CYCtarget gene transcription is at its maximum. Together with the finding that no periodic PER-TDT nuclear localization is observed in cultured clock neurons (Figure 2F), these results suggest that the feedback inhibition of CLK/CYC transcriptional activity by nuclear PER is impaired in isolated clock neurons, resulting in the constant upregulation of CLK/CYC-mediated transcription. Constant rise of $3 \times 69-\mathrm{VNP}$ levels in $w t$ dissociated neurons (Figure 1B) fits this model. Importantly, $3 \times 69-\mathrm{VNP}$ levels exhibit fluctuations but not a steady increase in $p d f r$ mutant cells in dispersed culture (Figure 4A). This observation rules out that the steady increase of $3 \times 69$-VNP levels in $w t$ dissociated neurons is caused by a general impairment of protein degradation systems. Furthermore, it indicates that CLK/CYC-mediated transcription sustains in a stochastic manner in dissociated neurons even in the absence of PDF signaling.

Cytoplasmic enrichment and the absence of rhythmic nuclear accumulation of PER suggest an impairment in nuclear translocation or the stability of nuclear PER. PER nuclear entry 


\section{A}

Endogenous PER/TIM levels

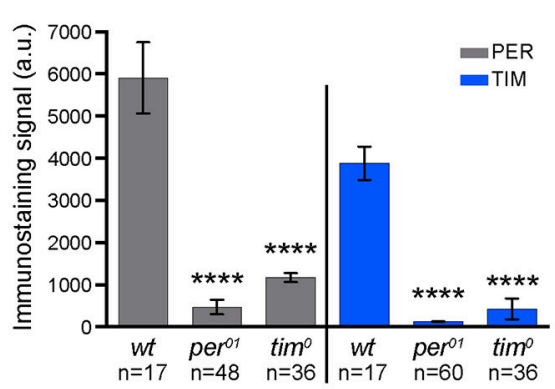

C

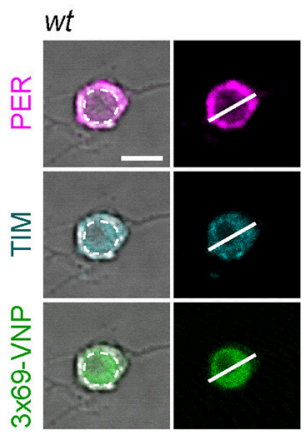

E

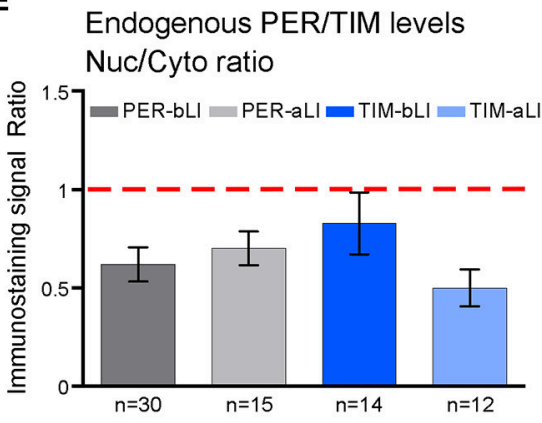

Endogenous PER/TIM levels

before or after Live Imaging

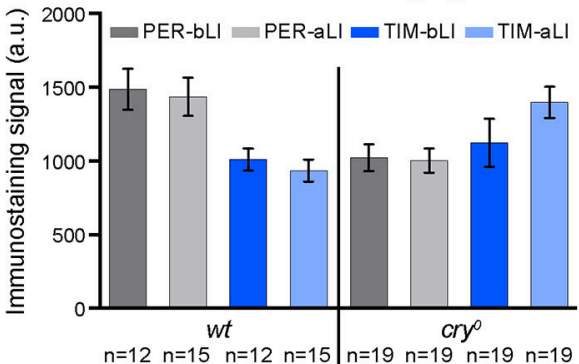

D
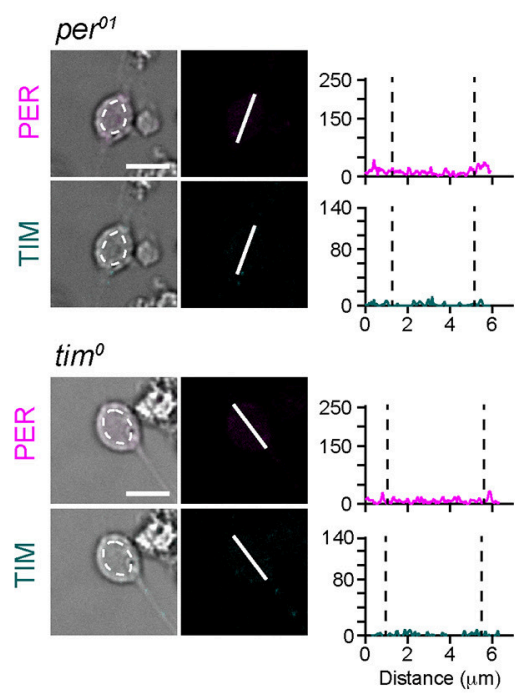

$\mathbf{F}$

PER-TDT levels Nuc/Cyto ratio

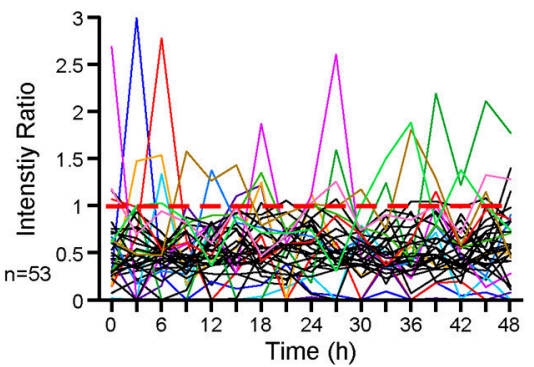

FIGURE 2 | PER and TIM are mainly cytoplasmic in dissociated cultured neurons. (A-E) The levels and subcellular localization of endogenous PER and TIM were analyzed by immunostaining. (A) wt clock neurons express PER and TIM ( ${ }^{\star \star \star \star} p<0.0001$ between $w t$ and per ${ }^{01}$ or tim ${ }^{0}$ by one-way ANOVA with Tukey's correction for multiple comparisons). (B) PER and TIM expression levels did not decrease after live imaging. bLI, before live imaging; aLI, after live imaging. Mean, error bars represent \pm SEM. The values between before and after live imaging were compared by one-way ANOVA with Tukey's correction for multiple comparisons and showed no significant differences. (C,D) Representative images of cultured neurons double stained for PER and TIM. Scale bar, $5 \mu \mathrm{m}$. (C) Clock neurons expressing $3 \times 69-$ VNP also express PER and TIM. The graphs represent the $z$-axis profiles of the fluorescence along the indicated line. Dotted lines in the graphs indicate the position of the nucleus, which was manually defined on the bright-field image (dotted circles on the left panels). Y-axes represent the fluorescence intensity in arbitrary unit. Z-axis profiles are plotted for the presentation purpose only but not for quantitative analysis. (D) Negative controls for anti-PER/TIM staining performed on the neuron derived from per01 or tim0 mutant larvae. (E) Nuclear/Cytoplasmic (Nuc/Cyto) ratio of PER and TIM staining signal before and after live imaging. Fluorescence intensity was measured using 3D image analysis. Mean \pm SEM. Comparison between aLI and bLI by Mann-Whitney test showed no significant differences.

(F) Nuc/Cyto ratio of PER-TDT fluorescence intensity of individual clock neurons during 48-h live imaging of wt clock neurons. For clarity, only the cells that showed nuclear enrichment (ratio > 1) are shown in color. 


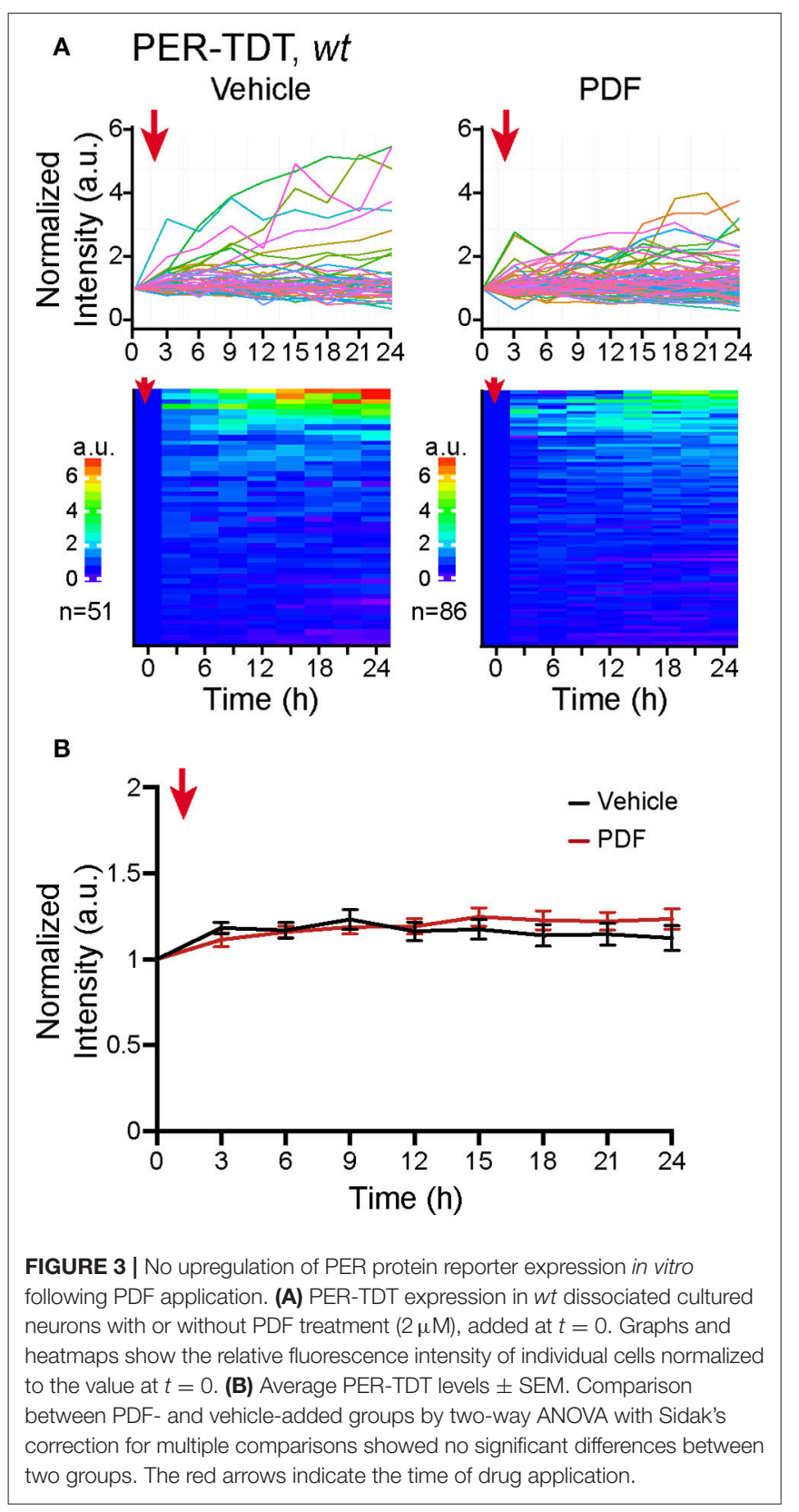

is controlled by multiple kinases, including CK2 (HelfrichForster, 2005; Chiu et al., 2008), SGG (Martinek et al., 2001), and probably a still unknown proline-directed kinase (Ko et al., 2010). PER phosphorylation by DBT promotes PER degradation, which is prevented by binding to TIM (Kloss et al., 1998; Ko et al., 2002; Kim et al., 2007). Phosphorylated PER enters the nucleus as PER/TIM dimer, where dephosphorylation by PP1 (Fang et al., 2007) and PP2A (Sathyanarayanan et al., 2004) prevents the phosphorylation at the sites promoting the binding of the E3 ubiquitin ligase SLIMB and eventual degradation (Chiu et al., 2008). Therefore, some of these post-translational mechanisms are probably dysregulated in the dispersed clock neurons.

\section{Network-Dependent Effect of PDF Signaling on the Molecular Clock}

Our results also suggest that signaling downstream of PDF/PDFR may work differently in isolated clock neurons, which exacerbates the dampening of the molecular rhythms. In brain explants during the night, PDF enhances CLK/CYCmediated transcription via neuronal activity-dependent but c-AMP independent mechanism. At the same time, PDF signaling stabilizes PER via cAMP-PKA dependent mechanism (Li et al., 2014; Seluzicki et al., 2014; Sabado et al., 2017). The latter mechanism seems to be inhibited in dispersed clock neurons, as the addition of PDF to the culture medium or $p d f r$ mutation did not affect PER-TDT expression profile (Figures 3, 4B). Thus, PER is probably less stable in dissociated neurons even in wt culture than in vivo, which also impairs negative feedback on CLK/CYC-mediated transcription.

We previously showed that the addition of PDF in the medium increases the $3 \times 69$-VNP levels in dissociated neurons, whereas PDF upregulates $3 \times 69$-VNP levels only during the night in brain explants (Sabado et al., 2017). Collectively, these results postulate that transcriptional response to $\mathrm{PDF}$ is negatively regulated during the day in the brain. In contrast, our results here indicate that PER levels are insensitive to PDF signaling in dissociated culture, whereas PDF stabilizes PER during the night in brain explants. Therefore, the nighttime-specific effect of PDF on PER stabilization probably occurs via a positive regulatory mechanism that happens only within the intact circadian circuit. These negative and positive gating of molecular clock response to PDF signaling requires cycling clocks, which appear to be the emergent property of the intact circadian circuitry (Supplementary Figure 3). Intriguingly, recent studies using in vivo $\mathrm{Ca}^{2+}$ imaging demonstrated that PDF controls the "morning" and "evening" phases of the $\mathrm{Ca}^{2+}$ rhythms in different subpopulation of pacemaker neurons, independent of their molecular phases (Liang et al., 2016, 2017). Thus, our results add to a growing body of evidence that PDF differentially regulates molecular and neuronal rhythms through complex and network-dependent mechanisms.

Of note, unlike the l-LNvs, the s-LNvs do not express the neuropeptide amidation enzyme PHM; hence, the PDF released from the s-LNvs might be either non-amidated or amidated by another amidation machinery (Helfrich-Forster, 2009, Taghert et al., 2001). Since larval LNvs are the precursors of adult s-LNvs, we used non-amidated PDF for all the pharmacological experiments in this and our previous study (Sabado et al., 2017). Non-amidated PDF can trigger PDFR-dependent, timeof-day- and activity-dependent response of molecular clockwork in cultured larval brains as well as TTX-sensitive CLK/CYCmediated transcription in cultured clock neurons (Sabado et al., 2017). Thus, although non-amidated neuropeptides have shorter half-lives and are less bioactive than the amidated ones, nonamidated PDF is bioactive at least in these assays. Nevertheless, since PDF secreted from larval LNvs and adult s-LNvs may as well be amidated (Taghert et al., 2001; Park et al., 2008), the results of these assays might not fully represent the native activity of 

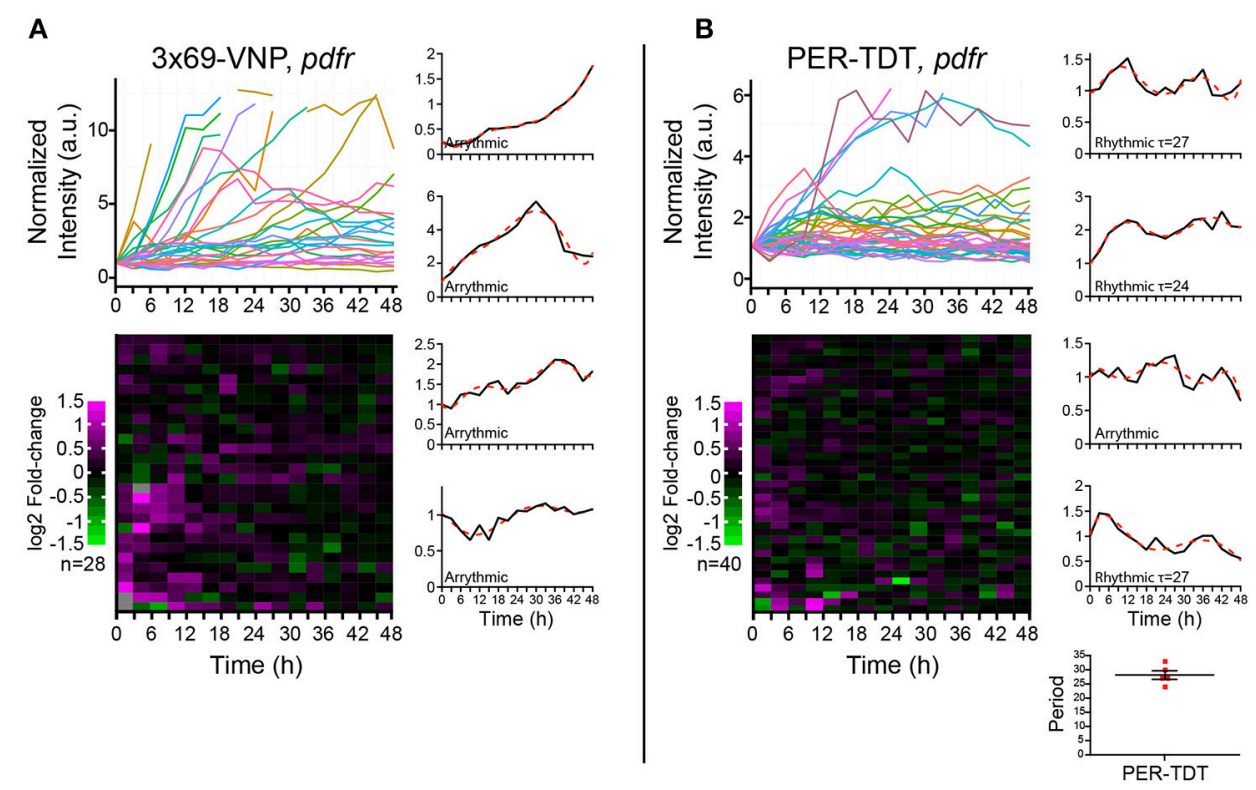

FIGURE 4 | Properties of the Drosophila neuronal clocks in the absence of PDF signaling. (A) 3×69-VNP and (B) PER-TDT expression levels in dissociated cultured neurons prepared from pdfr mutants, normalized to the value at $t=0$. Unlike in the wild-type culture, $3 \times 69-\mathrm{VNP}$ levels fluctuate in the pdfr mutant culture. Graphs and raster plots are as in Figure 1. Bottom right, periods of the neurons that showed circadian rhythms in PER-TDT levels (12.5\% of PER-TDT-expressing cells). Mean period \pm SEM was $28.2 \pm 1.53 \mathrm{~h}$. $\mathrm{R}$, rhythmic; AR, arrhythmic.

endogenous PDF. It will be important in future studies to validate whether non-amidated PDF can activate PDFR and also test the effect of amidated PDF in the same assays.

\section{Network-Driven Molecular Oscillations: A Conserved Feature of Neuronal Clocks?}

While no cells in dissociated culture exhibit circadian rhythms in $3 \times 69$-VNP expression, approximately one-tenth of clock neurons display PER-TDT fluctuations with periods in the circadian range (Figures 1, 4). These results suggest that, certain post-transcriptional mechanisms involved in PER protein stability or transcriptional regulation via per regulatory sequences other than the CRS is still functioning in dispersed clock neurons. These regulatory mechanisms prevent overaccumulation of PER and even produce PER protein oscillations in the circadian range in some cells. Our results thus showcase the emerging notion that post-transcriptional controls reinforce robustness of circadian oscillation (Lim and Allada, 2013).

A previous work in mammals has shown that, in lowdensity dispersed culture, only $\sim 18 \%$ of SCN neurons display circadian rhythms with periods ranging from 15 to $35 \mathrm{~h}$ (Webb et al., 2009). This is reminiscent of our observation that $\sim 13 \%$ of isolated fly clock neurons exhibited PER protein rhythms. Moreover, it is noteworthy that we did not detect obvious celltype specificity as to which neurons display circadian rhythms in dissociated culture. This implies that it is probabilistic rather than deterministic whether or not a neuron displays PER rhythms in isolation. Similarly, it was reported that isolated SCN neurons sustain or lose rhythmicity in random fashion (Webb et al., 2009). Thus, both Drosophila and SCN neurons seem to be poor oscillators that require network interactions to generate robust circadian rhythms. Whether or not a given pacemaker neuron sustain circadian molecular rhythms isolated from the intact circuit probably depends on the cell's physiological state, such as ion conductance and excitability. It has been proposed that the sensitivity of SCN neurons to the intercellular communication provides the circadian network the plasticity to promptly respond to changes in environmental conditions and produce a coherent output (Ko et al., 2010; Webb and Oates, 2016). It may also be a common principle of neuronal clocks in Drosophila and mammals.

\section{MATERIALS AND METHODS}

\section{Fly Strains}

Drosophila were reared at $25^{\circ} \mathrm{C}$ on a corn-meal medium under 12:12 h light-dark cycles (LD). The 1982clk-gal4 line (Gummadova et al., 2009), The GAL4 enhancer trap line gal1118 (Blanchardon et al., 2001), cry (Dolezelova et al., 2007), and $p d f r^{5304}$ (Hyun et al., 2005), UAS-mCD8::VENUS and UAS-mCD8::RFP, $3 \times 69$-Venus and per-TdT lines (Sabado et al., 2017) have been previously characterized.

\section{Primary Neuron Culture}

Brains of LD-entrained non-wandering L3 larvae were dissected in ice-cold saline solution (Jiang et al., 2005) at ZT11. Dissociated neuron culture was performed as previously described (KuppersMunther et al., 2004; Saad et al., 2012) with the following modifications. In brief, the dissected brains were enzymatically treated with $50 \mathrm{unit} / \mathrm{mL}$ papain (Worthington) and mechanically 
dissociated. The cell suspension was then plated on glassbottom dishes $(35 \mathrm{~mm}$ MatTek petri dish, $10 \mathrm{~mm}$ microwell with $0.16 / 0.19 \mathrm{~mm}$ coverglass) coated with Concanavalin A (Sigma). Approximately $3.1 \sim 4 \times 10^{5}$ cells, which corresponds to the suspension derived from ca. Ten larval brains, were plated in each dish $\left(4 \sim 5 \times 10^{5}\right.$ cells $\left./ \mathrm{cm}^{2}\right)$. Once the cells were attached to the glass bottom, the dish was flooded with SM $^{\text {active }}$ (Supplementary Table 1). The dissociated neuron culture was incubated at $25^{\circ} \mathrm{C}$ in $80 \%$ relative humidity and in constant darkness for 2 days prior to time-lapse imaging. Time-lapse imaging was carried out in the same culture conditions.

For pharmacological experiments, the neuropeptide PDF (custom-made by Chi Scientific, H-NSELINSLLSLPKNMNDA$\mathrm{OH} ; 2 \mu \mathrm{M}$ ) or the vehicle (DMSO) was added to the cell culture medium just before the start of the time-lapse imaging.

\section{Immunostaining of Cultured Neurons}

Anti-PER and Anti-TIM immunostaining of the cultured neurons was performed as previously described with some modifications (Nawathean et al., 2005). After 2 or 4 days in culture, the medium was discarded, and the culture was washed with phosphate-buffered saline (PBS); then, the cells were fixed with $1 \%$ paraformaldehyde in PBS for $20 \mathrm{~min}$ at room temperature. The cells were then washed with $0.1 \%$ Triton in PBS, and 1:1,000 rabbit anti-PER and 1:2,500 rat anti-TIM (gift from F. Rouyer) in blocking buffer were used as the primary antibodies. The secondary antibodies, anti-rabbit-Alexa633 and anti-rat-Alexa-555 (Invitrogen), were diluted 1:500 in the blocking buffer.

\section{Live Imaging}

A Leica TCS SP5 tandem scanner confocal microscope was used for fluorescence imaging. The same parameter settings were applied to image all the samples of the same type. Immunostained cultured neurons were scanned using a $\times 63$ water-immersion objective with the galvo scanner at $400 \mathrm{~Hz}$. For time-lapse imaging, $\times 63$ objective was used and the images were acquired every $3 \mathrm{~h}$ for $48 \mathrm{~h}$ using the resonant scanner at $8,000 \mathrm{~Hz}$ with high-sensitivity HyD detectors. The bi-directional scan was used together with $8 \times$ line average. A Z-section step of $1.7 \mu \mathrm{m}$ $\times 8$ was used for imaging dissociated cultured neurons. A $514 \mathrm{~nm}$ laser was used to excite VENUS fluorophore $(0.68$ $\mu \mathrm{W} / \mathrm{cm}^{2}$ to image VNP, $0.40 \mu \mathrm{W} / \mathrm{cm}^{2}$ for mCD8::VENUS), and a $561 \mathrm{~nm}$ laser was used for the TdTOMATO and mRFP fluorophores $\left(14.8 \mu \mathrm{W} / \mathrm{cm}^{2}\right.$ for PER-TDT, $5.71 \mu \mathrm{W} / \mathrm{cm}^{2}$ for mCD8::RFP). Laser intensity was measured at the level of the sample with a microscope slide power meter (Thorlabs, S170C).

\section{Image Analysis}

The fluorescence intensities in immunostained cultured neurons and the time-lapse movies of the cultured neurons were measured with the Imaris software (Bitplane). A 3D mask built from the fluorescent signal emitted by the clock neurons over the time course was created using the surface tool of Imaris. The same threshold settings, including background subtraction, were applied to all the samples of the same type. The intensity SUM was then extracted from the statistical data that were automatically generated by the program.

\section{Time Series Data Analysis}

Fluorescence intensity time series data were normalized to the value at $t=0$ except for the raster plots for intensity foldchange. Heatmaps representing the fluorescence intensity time course were generated with an in-house $\mathrm{R}$ script. Each row represents the data of a single cell. Rows were ordered by the highest intensity over $24 \mathrm{~h}$. To generate raster plots for intensity fold-change, the value at $t=\mathrm{n}+1$ was divided by the value of $t=\mathrm{n}$, and the $\log 2$ of the fold-change value was plotted over time using an in-house $\mathrm{R}$ script. The $\log 2$ foldchange values were color-coded, in which positive fold-changes are shown with a magenta gradient, no change is shown in black, and negative changes are shown with a green gradient. The hierarchical clustering analysis (complete linkage method) was performed to find similar clusters and visualize the plots accordingly.

For rhythm analysis, a combination of manual inspection and Maximum Entropy Spectral Analysis (MESA) was used to detect rhythmicity and estimate the period. The data of the entire recording period $(48 \mathrm{~h})$ were subjected to the analysis. MESA analysis was performed using an in-house $\mathrm{R}$ script containing the spec.ar function. MESA was chosen because, unlike other methods, it is adapted for the detection of the rhythms in short or noisy time series (Dowse et al., 1989; Levine et al., 2002; Klarsfeld et al., 2003). First, the intensity time course plots were generated using Excel or Prism (GraphPad Prism version 6.0c for Mac, GraphPad Software, San Diego California USA, www. graphpad.com). Because we found that 6th-order polynomial regression models best fit the data, we superimposed the 6thorder polynomial trend lines to the graphs to facilitate the detection of rhythmicity. Then, the time series data that have circadian rhythms, defined by the peak-to-peak interval from 18 to $36 \mathrm{~h}$, were identified by manual inspection. However, the polynomial trend lines were not used for determining the period. In parallel, the intensity time series data without normalization were analyzed with MESA without filtering. The time series data were scored as circadian only when both the manual and MESA analyses detected the rhythms with a period between 18 and $36 \mathrm{~h}$.

\section{Statistical Analysis}

Statistical analyses were performed with GraphPadPrism software to compare the expression of antibodies or transgenes in different genetic backgrounds or pharmacological conditions. Non-parametric tests were used when the data did not follow a normal distribution. To compare the effect of one condition (genetic backgrounds or pharmacological conditions) parametric or non-parametric $T$-Tests or one-way ANOVA with a correction for multiple comparisons (depending on the number of data sets to compare) were used. For live imaging experiments, time was an added condition, hence, two-way 
ANOVA with a correction for multiple comparisons were utilized. Details of the used statistical tests are given in the figure legends.

\section{AUTHOR CONTRIBUTIONS}

VS and EN designed the work. VS and LV performed the experiments and analyzed the data. VS, LV, and EN wrote the manuscript.

\section{FUNDING}

This work was supported by the IST PRESTO program, the Swiss National Science Foundation (31003A_149893 and 31003A_169548), the European Research Council
(ERC-StG-311194), Novartis Foundation for MedicalBiomedical Research (13A39) and the University of Geneva.

\section{ACKNOWLEDGMENTS}

We thank F. Rouyer for antibodies and Bloomington Drosophila Stock Center for fly stocks. We also thank Jose Manuel Nunes for helping data analysis.

\section{SUPPLEMENTARY MATERIAL}

The Supplementary Material for this article can be found online at: https://www.frontiersin.org/articles/10.3389/fncel. 2017.00317/full\#supplementary-material

\section{REFERENCES}

Beckwith, E. J., and Ceriani, M. F. (2015). Communication between circadian clusters: the key to a plastic network. FEBS Lett. 589, 3336-3342. doi: 10.1016/j.febslet.2015.08.017

Blanchardon, E., Grima, B., Klarsfeld, A., Chelot, E., Hardin, P. E., Préat, T., et al. (2001). Defining the role of Drosophila lateral neurons in the control of circadian rhythms in motor activity and eclosion by targeted genetic ablation and PERIOD protein overexpression. Eur. J. Neurosci. 13, 871-888. doi: 10.1046/j.0953-816x.2000.01450.x

Chiu, J. C., Vanselow, J. T., Kramer, A., and Edery, I. (2008). The phospho-occupancy of an atypical SLIMB-binding site on PERIOD that is phosphorylated by DOUBLETIME controls the pace of the clock. Genes Dev. 22, 1758-1772. doi: 10.1101/gad.1682708

Dolezelova, E., Dolezel, D., and Hall, J. C. (2007). Rhythm defects caused by newly engineered null mutations in Drosophila's cryptochrome gene. Genetics 177, 329-345. doi: 10.1534/genetics.107.076513

Dowse, H. B., Dushay, M. S., Hall, J. C., and Ringo, J. M. (1989). Highresolution analysis of locomotor activity rhythms in disconnected, a visualsystem mutant of Drosophila melanogaster. Behav. Genet. 19, 529-542. doi: 10.1007/BF01066252

Emery, P., So, W. V., Kaneko, M., Hall, J. C., and Rosbash, M. (1998). CRY, a Drosophila clock and light-regulated cryptochrome, is a major contributor to circadian rhythm resetting and photosensitivity. Cell 95, 669-679. doi: 10.1016/S0092-8674(00)81637-2

Fang, Y., Sathyanarayanan, S., and Sehgal, A. (2007). Post-translational regulation of the Drosophila circadian clock requires protein phosphatase 1 (PP1). Genes Dev. 21, 1506-1518. doi: 10.1101/gad.1541607

Gorostiza, E. A., and Ceriani, M. F. (2013). Retrograde bone morphogenetic protein signaling shapes a key circadian pacemaker circuit. J. Neurosci. 33, 687-696. doi: 10.1523/JNEUROSCI.3448-12.2013

Granados-Fuentes, D., and Herzog, E. D. (2013). The clock shop: coupled circadian oscillators. Exp. Neurol. 243, 21-27. doi: 10.1016/j.expneurol.2012.10.011

Gummadova, J. O., Coutts, G. A., and Glossop, N. R. (2009). Analysis of the Drosophila Clock promoter reveals heterogeneity in expression between subgroups of central oscillator cells and identifies a novel enhancer region. J. Biol. Rhythms 24, 353-367. doi: 10.1177/0748730409343890

Hao, H., Allen, D. L., and Hardin, P. E. (1997). A circadian enhancer mediates PER-dependent mRNA cycling in Drosophila melanogaster. Mol. Cell Biol. 17, 3687-3693. doi: 10.1128/MCB.17.7.3687

Hao, H., Glossop, N. R., Lyons, L., Qiu, J., Morrish, B., Cheng, Y., et al. (1999). The 69 bp circadian regulatory sequence (CRS) mediates per-like developmental, spatial, and circadian expression and behavioral rescue in Drosophila. J. Neurosci. 19, 987-994.

Hardin, P. E. (2011). Molecular genetic analysis of circadian timekeeping in Drosophila. Adv. Genet. 74, 141-173. doi: 10.1016/B978-0-12387690-4.00005-2

Hardin, P. E., and Panda, S. (2013). Circadian timekeeping and output mechanisms in animals. Curr. Opin. Neurobiol. 23, 724-731. doi: 10.1016/j.conb.2013.02.018

Helfrich-Forster, C. (2005). Neurobiology of the fruit fly's circadian clock. Genes Brain Behav. 4, 65-76. doi: 10.1111/j.1601-183X.2004.00092.x

Helfrich-Forster, C. (2009). Neuropeptide PDF plays multiple roles in the circadian clock of Drosophila melanogaster. Sleep Biol. Rhythms 7, 130-143. doi: 10.1111/j.1479-8425.2009.00408.x

Hyun, S., Lee, Y., Hong, S. T., Bang, S., Paik, D., Kang, J., et al. (2005). Drosophila GPCR Han is a receptor for the circadian clock neuropeptide PDF. Neuron 48, 267-278. doi: 10.1016/j.neuron.2005.08.025

Jiang, S. A., Campusano, J. M., Su, H., and O’Dowd, D. K. (2005). Drosophila mushroom body Kenyon cells generate spontaneous calcium transients mediated by PLTX-sensitive calcium channels. J. Neurophysiol. 94, 491-500. doi: 10.1152/jn.00096.2005

Kim, E. Y., Ko, H. W., Yu, W., Hardin, P. E., and Edery, I. (2007). A DOUBLETIME kinase binding domain on the Drosophila PERIOD protein is essential for its hyperphosphorylation, transcriptional repression, and circadian clock function. Mol. Cell Biol. 27, 5014-5028. doi: 10.1128/MCB.02339-06

Klarsfeld, A., Leloup, J. C., and Rouyer, F. (2003). Circadian rhythms of locomotor activity in Drosophila. Behav. Process. 64, 161-175. doi: 10.1016/S0376-6357(03)00133-5

Kloss, B., Price, J. L., Saez, L., Blau, J., Rothenfluh, A., Wesley, C. S., et al. (1998). The Drosophila clock gene double-time encodes a protein closely related to human casein kinase Iepsilon. Cell 94, 97-107. doi: 10.1016/S0092-8674(00)81225-8

Ko, C. H., Yamada, Y. R., Welsh, D. K., Buhr, E. D., Liu, A. C., Zhang, E. E., et al. (2010). Emergence of noise-induced oscillations in the central circadian pacemaker. PLoS Biol 8:e1000513. doi: 10.1371/journal.pbio.1000513

Ko, H. W., Jiang, J., and Edery, I. (2002). Role for Slimb in the degradation of Drosophila Period protein phosphorylated by Doubletime. Nature 420, 673-678. doi: 10.1038/nature01272

Kuppers-Munther, B., Letzkus, J. J., Luer, K., Technau, G., Schmidt, H., and Prokop, A. (2004). A new culturing strategy optimises Drosophila primary cell cultures for structural and functional analyses. Dev. Biol. 269, 459-478. doi: 10.1016/j.ydbio.2004.01.038

Lear, B. C., Merrill, C. E., Lin, J. M., Schroeder, A., Zhang, L., and Allada, R. (2005). A G protein-coupled receptor, groom-of-PDF, is required for PDF neuron action in circadian behavior. Neuron 48, 221-227. doi: 10.1016/j.neuron.2005.09.008

Levine, J. D., Funes, P., Dowse, H. B., and Hall, J. C. (2002). Signal analysis of behavioral and molecular cycles. BMC Neurosci 3:1. doi: 10.1186/1471-2202-3-1

Li, Y., Guo, F., Shen, J., and Rosbash, M. (2014). PDF and cAMP enhance PER stability in Drosophila clock neurons. Proc. Natl. Acad. Sci. U.S.A. 111, E1284-E1290. doi: 10.1073/pnas.1402562111

Liang, X., Holy, T. E., and Taghert, P. H. (2016). Synchronous Drosophila circadian pacemakers display nonsynchronous $\mathrm{Ca}(2)(+)$ rhythms in vivo. Science 351, 976-981. doi: 10.1126/science.aad3997 
Liang, X., Holy, T. E., and Taghert, P. H. (2017). A series of suppressive signals within the Drosophila circadian neural circuit generates sequential daily outputs. Neuron 94, 1173. e4-1189.e4. doi: 10.1016/j.neuron.2017.05.007

Lim, C., and Allada, R. (2013). Emerging roles for post-transcriptional regulation in circadian clocks. Nat. Neurosci. 16, 1544-1550. doi: 10.1038/nn.3543

Liu, A. C., Welsh, D. K., Ko, C. H., Tran, H. G., Zhang, E. E., Priest, A. A., et al. (2007). Intercellular coupling confers robustness against mutations in the SCN circadian clock network. Cell 129, 605-616. doi: 10.1016/j.cell.2007.02.047

Malpel, S., Klarsfeld, A., and Rouyer, F. (2004). Circadian synchronization and rhythmicity in larval photoperception-defective mutants of Drosophila. J. Biol. Rhythms 19, 10-21. doi: 10.1177/0748730403260621

Martinek, S., Inonog, S., Manoukian, A. S., and Young, M. W. (2001). A role for the segment polarity gene shaggy/GSK-3 in the Drosophila circadian clock. Cell 105, 769-779. doi: 10.1016/S0092-8674(01)00383-X

Mazzoni, E. O., Desplan, C., and Blau, J. (2005). Circadian pacemaker neurons transmit and modulate visual information to control a rapid behavioral response. Neuron 45, 293-300. doi: 10.1016/j.neuron.2004.12.038

Mertens, I., Vandingenen, A., Johnson, E. C., Shafer, O. T., Li, W., Trigg, J. S., et al. (2005). PDF receptor signaling in Drosophila contributes to both circadian and geotactic behaviors. Neuron 48, 213-219. doi: 10.1016/j.neuron.2005.09.009

Nagoshi, E., Saini, C., Bauer, C., Laroche, T., Naef, F., and Schibler, U. (2004). Circadian gene expression in individual fibroblasts: cell-autonomous and self-sustained oscillators pass time to daughter cells. Cell 119, 693-705. doi: 10.1016/j.cell.2004.11.015

Nawathean, P., Menet, J. S., and Rosbash, M. (2005). Assaying the Drosophila negative feedback loop with RNA interference in S2 cells. Methods Enzymol. 393, 610-622. doi: 10.1016/S0076-6879(05)93032-2

Park, D., Veenstra, J. A., Park, J. H., and Taghert, P. H. (2008). Mapping peptidergic cells in Drosophila: where DIMM fits in. PLoS ONE 3:e1896. doi: 10.1371/journal.pone.0001896

Peng, Y., Stoleru, D., Levine, J. D., Hall, J. C., and Rosbash, M. (2003). Drosophila free-running rhythms require intercellular communication. PLoS Biol. 1:E13. doi: 10.1371/journal.pbio.0000013

Roberts, L., Leise, T. L., Noguchi, T., Galschiodt, A. M., Houl, J. H., Welsh, D. K., et al. (2015). Light evokes rapid circadian network oscillator desynchrony followed by gradual phase retuning of synchrony. Curr. Biol. 25, 858-867. doi: 10.1016/j.cub.2015.01.056

Saad, Y., Anabosi, M., Anava, S., Nadav, G., Yerushalmi, Y., and Ayali, A. (2012). Fly neurons in culture: a model for neural development and pathology. J. Mol. Histol. 43, 421-430. doi: 10.1007/s10735-012-9417-z

Sabado, V., Vienne, L., Nunes, J. M., Rosbash, M., and Nagoshi, E. (2017). Fluorescence circadian imaging reveals a PDF-dependent transcriptional regulation of the Drosophila molecular clock. Sci. Rep. 7:41560. doi: $10.1038 /$ srep 41560

Sathyanarayanan, S., Zheng, X., Xiao, R., and Sehgal, A. (2004). Posttranslational regulation of Drosophila PERIOD protein by protein phosphatase 2A. Cell 116, 603-615. doi: 10.1016/S0092-8674(04)00128-X

Sellix, M. T., Currie, J., Menaker, M., and Wijnen, H. (2010). Fluorescence/luminescence circadian imaging of complex tissues at single-cell resolution. J. Biol. Rhythms 25, 228-232. doi: 10.1177/0748730410368016

Seluzicki, A., Flourakis, M., Kula-Eversole, E., Zhang, L., Kilman, V., and Allada, R. (2014). Dual PDF signaling pathways reset clocks via TIMELESS and acutely excite target neurons to control circadian behavior. PLoS Biol. 12:e1001810. doi: 10.1371/journal.pbio.1001810

Sheeba, V., Kaneko, M., Sharma, V. K., and Holmes, T. C. (2008). The Drosophila circadian pacemaker circuit: Pas de deux or tarantella? Crit. Rev. Biochem. Mol. Biol. 43, 37-61. doi: 10.1080/10409230701829128

Stanewsky, R., Kaneko, M., Emery, P., Beretta, B., Wager-Smith, K., Kay, S., et al. (1998). The cryb mutation identifies cryptochrome as a circadian photoreceptor in Drosophila. Cell 95, 681-692. doi: 10.1016/S0092-8674(00)81638-4

Taghert, P. H., Hewes, R. S., Park, J. H., O’Brien, M. A., Han, M., and Peck, M. E. (2001). Multiple amidated neuropeptides are required for normal circadian locomotor rhythms in Drosophila. J. Neurosci. 21, 6673-6686.

Webb, A. B., and Oates, A. C. (2016). Timing by rhythms: daily clocks and developmental rulers. Dev. Growth Differ 58, 43-58. doi: 10.1111/dgd.12242

Webb, A. B., Angelo, N., Huettner, J. E., and Herzog, E. D. (2009). Intrinsic, nondeterministic circadian rhythm generation in identified mammalian neurons. Proc. Natl. Acad. Sci. U.S.A. 106, 16493-16498. doi: 10.1073/pnas.0902768106

Welsh, D. K., Yoo, S. H., Liu, A. C., Takahashi, J. S., and Kay, S. A. (2004). Bioluminescence imaging of individual fibroblasts reveals persistent, independently phased circadian rhythms of clock gene expression. Curr. Biol. 14, 2289-2295. doi: 10.1016/j.cub.2004.11.057

Yamaguchi, S., Isejima, H., Matsuo, T., Okura, R., Yagita, K., Kobayashi, M., et al. (2003). Synchronization of cellular clocks in the suprachiasmatic nucleus. Science 302, 1408-1412. doi: 10.1126/science.1089287

Yao, Z., and Shafer, O. T. (2014). The Drosophila circadian clock is a variably coupled network of multiple peptidergic units. Science 343, 1516-1520. doi: 10.1126/science. 1251285

Yoo, S. H., Yamazaki, S., Lowrey, P. L., Shimomura, K., Ko, C. H., Buhr, E. D., et al. (2004). PERIOD2::LUCIFERASE real-time reporting of circadian dynamics reveals persistent circadian oscillations in mouse peripheral tissues. Proc. Natl. Acad. Sci. U.S.A. 101, 5339-5346. doi: 10.1073/pnas.0308709101

Yoshii, T., Hermann-Luibl, C., and Helfrich-Forster, C. (2016). Circadian light-input pathways in Drosophila. Commun. Integr. Biol. 9:e1102805. doi: 10.1080/19420889.2015.1102805

Yoshii, T., Wulbeck, C., Sehadova, H., Veleri, S., Bichler, D., Stanewsky, R., et al. (2009). The neuropeptide pigment-dispersing factor adjusts period and phase of Drosophila's clock. J. Neurosci. 29, 2597-2610. doi: 10.1523/JNEUROSCI.5439-08.2009

Zhang, Y., and Emery, P. (2012). "15: Molecular and Neural Control of Insect Circadian Rhythms A2," in Insect Molecular Biology and Biochemistry, ed L. I. Gilbert (San Diego, CA: Academic Press), 513-551.

Conflict of Interest Statement: The authors declare that the research was conducted in the absence of any commercial or financial relationships that could be construed as a potential conflict of interest.

Copyright (c) 2017 Sabado, Vienne and Nagoshi. This is an open-access article distributed under the terms of the Creative Commons Attribution License (CC BY). The use, distribution or reproduction in other forums is permitted, provided the original author(s) or licensor are credited and that the original publication in this journal is cited, in accordance with accepted academic practice. No use, distribution or reproduction is permitted which does not comply with these terms. 